\title{
Psychologie cognitive expérimentale
}

\section{Stanislas Dehaene}

\section{OpenEdition}

Journals

Édition électronique

URL : https://journals.openedition.org/annuaire-cdf/15303

DOI : 10.4000/annuaire-cdf.15303

ISBN : 978-2-7226-0572-5

ISSN : 2109-9227

Éditeur

Collège de France

Édition imprimée

Date de publication : 30 décembre 2020

Pagination : 157-165

ISBN : 978-2-7226-0516-9

ISSN : 0069-5580

Référence électronique

Stanislas Dehaene, "Psychologie cognitive expérimentale », L'annuaire du Collège de France [En ligne], 118 | 2020, mis en ligne le 01 avril 2021, consulté le 22 août 2022. URL : http://

journals.openedition.org/annuaire-cdf/15303 ; DOI : https://doi.org/10.4000/annuaire-cdf.15303 


\title{
PSYCHOLOGIE COGNITIVE EXPÉRIMENTALE
}

\author{
Stanislas DEHAENE \\ Membre de l'Institut (Académie des sciences), \\ professeur au Collège de France
}

Mots-clés : cerveau, comportement, langage, musique, évolution

Les séries de cours «Origines du langage et singularité de l'espèce humaine » et de séminaires "Modèles théoriques des représentations linguistiques et de leur évolution » sont disponibles en audio et/ou en vidéo, sur le site internet du Collège de France (https://www.college-de-france.fr/site/stanislas-dehaene/course-2017-2018. htm et https://www.college-de-france.fr/site/stanislas-dehaene/seminar-2017-2018. htm), ainsi que le colloque " Le rôle de l'expérimentation dans le domaine éducatif » (https://www.college-de-france.fr/site/stanislas-dehaene/symposium-2017-2018.htm).

\section{ENSEIGNEMENT}

\section{COURS - ORIGINES DU LANGAGE ET SINGULARITÉ DE L'ESPĖCE HUMAINE}

L'espèce humaine présente une multitude de spécificités cognitives qui la distinguent des autres animaux, au point qu'on peut légitimement parler d'une «singularité de l'espèce humaine » - sans pour autant nier, bien entendu, que chacune de nos facultés, comme le soulignait déjà Charles Darwin dans $L a$ Descendance de l'homme, trouve son origine dans l'évolution, donc dans l'existence de précurseurs toujours présents chez d'autres espèces animales.

La liste des singularités potentielles de l'espèce humaine est longue : usage de l'outil ? conscience de soi ? conscience des autres, par le biais de la théorie de l'esprit ? capacité de s'éduquer et d'enseigner aux autres ? créativité musicale, mathématique ou scientifique ? L'objectif du cours de cette année était d'examiner une seule hypothèse unificatrice, celle que la faculté de langage constitue le cœur de la singularité humaine. Selon cette hypothèse, nous posséderions, plus que tout autre espèce animale, des architectures neurales qui nous rendent capables d'exprimer nos pensées de façon symbolique et de les partager avec d'autres. 


\section{Cours 1 - Introduction aux hypothèses sur la singularité de l'espèce humaine}

Dans le premier cours, nous avons passé en revue les diverses hypothèses qui ont été proposées sur les fonctions cognitives qui constituent le noyau de la faculté de langage. En partant des propositions systématiques mais déjà anciennes du linguiste James Hockett, et des idées plus récentes de l'article clé de Mark Hauser, Noam Chomsky et W. Tecumseh Fitch (2002), nous avons retenu deux hypothèses essentielles :

- premièrement, le cerveau humain posséderait une capacité singulière de se forger un système de liens symboliques, arbitraires, bidirectionnels, reliant des signifiants et signifiés (selon la terminologie saussurienne);

- deuxièmement, le cerveau humain disposerait d'un ou plusieurs systèmes combinatoires permettant de composer ces symboles entre eux afin de produire une infinité d'expressions capables d'évoquer une infinité de sens.

Ces deux hypothèses placent la singularité humaine à un haut niveau cognitif : la capacité de manipuler et de combiner des symboles. Dans ce premier cours, nous avons également abordé une autre hypothèse de plus bas niveau, sensori-motrice, selon laquelle la faculté de langage de l'espèce humaine trouverait son origine dans l'anatomie et la biomécanique de l'appareil vocal. Les recherches récentes suggèrent que les modifications de l'anatomie du larynx ne jouent peut-être pas un rôle aussi essentiel qu'on le pensait autrefois, dans la mesure où deux études indiquent que l'appareil vocal des macaques et des babouins est déjà capable de produire de nombreux phonèmes. En revanche, dans les circuits cérébraux de contrôle de l'appareil vocal, on voit clairement apparaître des adaptations propres à Homo sapiens: l'existence d'un cortex moteur laryngé spécifique et de projections axonales directes vers les neurones moteurs du tronc cérébral.

\section{Cours 2 - La communication animale spontanée et ses limites}

Dans le deuxième cours, nous avons examiné les capacités de communication vocale que l'on observe spontanément chez différentes espèces animales. Chez le primate non humain, ces capacités sont limitées à la production de cris d'alarme qui font référence aux différents types de prédateurs rencontrés. Seule une syntaxe rudimentaire a été rapportée, par exemple chez les singes de Campell. La capacité de composer des messages complexes et intentionnels semble totalement absente, et les parallèles avec le langage humain sont donc très limités.

Tel n'est pas le cas chez l'oiseau chanteur. Comme le faisait déjà remarquer Darwin, les oiseaux doivent apprendre à chanter, et cet apprentissage suit des étapes de babillage et de convergence progressive vers les «mots » cibles qui présentent des parallèles étroits avec l'apprentissage de la phonologie et du vocabulaire chez l'enfant. Les mécanismes neuronaux de la production vocale, et même la génétique de ces réseaux cérébraux, sont remarquablement parallèles chez l'oiseau et dans le cerveau humain. En revanche, au niveau syntaxique, il n'y a - pour l'instant ? guère de preuve qu'aucune espèce d'oiseau ne soit capable de produire des phrases composées, organisées selon une syntaxe comparable à celle des langues humaines. Seules quelques relations complexes de dépendance à longue distance ont été rapportées chez le canari, et une forme de combinatoire sémantique fondée sur le nombre de syllabes chez la mésange à tête noire. En conclusion, à part le langage humain, aucun système spontané de communication animale ne semble faire appel à une syntaxe complexe pour faire référence à des contenus combinatoires. 


\section{Cours 3 - L'apprentissage de symboles chez l'animal}

Dans le troisième cours, nous avons examiné les études qui ont tenté d'enseigner des symboles et un langage, le plus souvent artificiel, à des primates non humains. Nous nous sommes appuyés sur une classification due à Charles Sanders Peirce, et revisitée par Terence Deacon et par Andreas Nieder, qui distingue plusieurs types de signes, c'est-à-dire de relations signifiant/signifié :

- les signes «iconiques », pour lesquels il existe une relation non arbitraire entre signifiant et signifié ;

- les signes « indexicaux », pour lesquels il existe une correspondance entre chaque signe arbitraire isolé et son référent, mais pas de système combinatoire dans lesquels ces signes viennent s'intégrer;

- enfin, les authentiques «symboles », c'est-à-dire des signes reliés entre eux par un vaste système combinatoire de relations. Dans la mesure où ces relations entre signifiants sont isomorphes avec celles qui relient les signifiés eux-mêmes (les contenus mentaux), il devient possible, par simple manipulation symbolique, de réaliser des opérations mentales qui conservent une correspondance systématique avec la réalité. L'exemple type est la correspondance entre les symboles des chiffres arabes et les quantités correspondantes : des calculs arithmétiques purement formels permettent d'anticiper sur les changements de quantités.

Dans ce cadre, les études expérimentales démontrent que de nombreux animaux (primates, perroquets, chiens, etc.) peuvent acquérir plusieurs centaines de signes arbitraires, atteignant ainsi le niveau 2 de Pierce. En revanche, ces signes acquis fonctionnent plutôt comme des indices que comme des symboles. En effet, ils présentent deux différences majeures avec les symboles des langues ou des systèmes sémiotiques de l'espèce humaine. D'une part, ils présentent une absence de réversibilité : l'animal ne comprend pas spontanément que la relation signifiant/ signifié est réversible, et qu'il est donc possible de passer du sens au symbole et vice versa, dans les deux directions. D'autre part, aucune espèce animale ne s'est avérée capable d'apprendre un authentique système de règles complexes liant les signes entre eux.

\section{Cours 4 - L'apprentissage de grammaires artificielles : études comportementales}

Dans le quatrième cours, nous sommes revenus sur les études comportementales qui ont tenté d'inculquer, à des primates ou à un perroquet, un système symbolique combinatoire, même rudimentaire. L'analyse démontre que les compétences de ces animaux restent très limitées dans le domaine syntaxique. Leur apprentissage semble fondé sur la mémoire de combinaisons spécifiques de mots ou de symboles, plutôt que sur une authentique grammaire générative. Le contraste avec l'espèce humaine est frappant : que ce soit dans le domaine des mots ou dans celui des règles logiques, les humains, y compris les jeunes enfants, apprennent rapidement à découvrir les règles générales qui régissent un domaine, une compétence qui excède celle des singes macaques ou des chimpanzés.

Dans un article de revue publié dans Neuron, nous avons proposé une classification en cinq niveaux de la représentation mentale des séquences: connaissance des transitions temporelles d'un item à l'autre (chaînes de Markov), formation de groupes (chunks), codage de l'ordre numérique, schémas algébriques abstraits (par 
exemple $x x Y$, une répétition suivie d'un item différent) et enfin structures arborescentes enchâssées, typiques des langues humaines (phrases formées de syntagmes enchâssés de façon récursive). L'analyse suggère que les quatre premiers niveaux sont accessibles aux autres espèces animales telles que le macaque ou même le rat. En revanche, il n'y a pas de preuve, au moins pour l'instant, que le cinquième niveau soit accessible à d'autres animaux que le primate humain.

\section{Cours 5 - L'apprentissage de grammaires artificielles: la saga du langage " $A^{n} B^{n}$ » et de ses variantes}

La théorie des langages formels, initiée par Noam Chomsky, introduit une différence fondamentale entre les grammaires à états finis, et celles dépendantes du contexte : seules ces dernières sont capables de représenter des structures enchâssées, récursives, qui sous-tendent la représentation mentale des langues humaines. L'hypothèse de Hauser, Chomsky et Fitch est que seule notre espèce est capable de récursion. De nombreux travaux expérimentaux ont tenté de réfuter ce postulat en tentant d'apprendre, à diverses espèces d'oiseaux chanteurs, une grammaire artificielle dite « $\mathrm{A}^{\mathrm{n}} \mathrm{B}^{\mathrm{n}}$ », c'est-à-dire une série de $n$ sons $\mathrm{A}$ suivis de $n$ sons $\mathrm{B}$. Cette grammaire présente l'intérêt de nécessiter une grammaire dépendante du contexte, et son apprentissage, dans l'espèce humaine, semble faire intervenir l'aire de Broca. Certains ont prétendu que les oiseaux chanteurs pouvaient apprendre ce type de grammaire, mais de nombreux arguments aussi bien théoriques que pratiques suggèrent que ces recherches ne sont pas convaincantes: le comportement des animaux est souvent peu spécifique, insuffisamment évalué, sans analyse des performances individuelles, et trop d'interprétations alternatives demeurent tenables.

Récemment, cependant, avec une approche très différente, l'équipe de Liping Wang et moi-même avons démontré que les singes macaques sont capables d'apprendre une grammaire "en miroir», avec des séquences de type ABBA, $\mathrm{ABCCBA}$, etc., où la seconde partie répète la première en miroir temporel. Ils y parviennent dans un contexte visuo-spatial et non auditif : leur mémoire spatiale leur permet de répéter une séquence en avant comme en arrière. Ainsi, le cerveau du singe macaque possède la capacité d'apprendre un langage supra-régulier, une grammaire indépendante du contexte (mais pas forcément toutes ces grammaires). Au strict minimum, la résolution de cette tâche nécessite une pile «last in, first out ». La singularité de l'espèce humaine pourrait résider dans la vitesse avec laquelle ce type de règle est découvert: les jeunes enfants d'âge préscolaire découvrent la règle en cinq essais maximum, et l'appliquent ensuite presque parfaitement, alors qu'il faut plusieurs dizaines de milliers d'essais aux singes macaques pour parvenir à une performance imparfaite. Le cerveau humain semble donc biaisé vers l'apprentissage rapide de règles et de structures enchâssées.

\section{Cours 6 - Codage cérébral des séquences auditives : l'imagerie cérébrale comparative et le paradigme local-global}

Dans le dernier cours, nous avons examiné quelles aires cérébrales, chez l'homme comme chez le singe macaque, contribuent à l'apprentissage de séquences auditives qui forment des grammaires élémentaires mais qui présentent une analogie partielle avec l'organisation du langage parlé. Les travaux comparatifs de Chris Petkov, fondés notamment sur les réponses aux violations d'une séquence auditive apprise, 
l'amènent à conclure qu'il existe un mécanisme de représentation cérébrale des séquences partagé par les deux espèces et hautement conservé dans l'évolution. C'est possible, mais nos propres travaux, fondés sur le paradigme local-global développé par Bekinschtein et al. (PNAS, 2009), suggèrent une différence majeure : dans l'espèce humaine, la région de Broca (gyrus frontal inférieur gauche, aires 44 et 45 de Brodmann) présente des réponses uniques d'intégration de plusieurs aspects de la séquence. Nous proposons que cette région soit capable de représenter les séquences sous la forme d'une formule compacte qui comprime toute l'information en un seul arbre symbolique récursif.

En résumé, l'acquisition du langage chez l'homme repose sur des circuits très spécifiques, notamment le cortex préfrontal inférieur et le sillon temporal supérieur de l'hémisphère gauche. Des circuits analogues existent chez les autres primates, mais ils ont subi une expansion considérable de leur surface et de leur connectivité au cours de l'hominisation. De plus, dans notre espèce, ces circuits intègrent plus d'informations que chez le singe macaque (travaux de Liping Wang). Si les primates non humains sont capables d'apprendre des signes arbitraires (associations signifiant/signifié), ils le font sans réversibilité ni construction mentale d'un vaste système de symboles. Ils parviennent à apprendre des séquences arbitraires, mais présentent des difficultés ou, au minimum, une lenteur considérable dans l'apprentissage de règles systématiques ou de grammaires complexes avec enchâssement récursif. Même si les animaux doués d'apprentissage vocal, comme les oiseaux chanteurs, présentent des circuits remarquablement analogues à ceux de l'espèce humaine, y compris sur le plan génétique, leur « langage » ne présente pas de structures enchâssées similaires à celles de notre espèce.

En ce qui concerne l'acquisition du langage et des symboles, notre espèce est donc bien singulière... La compréhension de l'origine neurobiologique de cette singularité est l'une des grandes questions qui se pose aux chercheurs pour les années à venir.

\section{SÉMINAIRES - MODĖLES THÉORIQUES DES REPRÉSENTATIONS LINGUISTIQUES ET DE LEUR ÉVOLUTION}

Nous avons reçu six invités spécialistes de la modélisation, à différents niveaux, des représentations du langage. Le séminaire a permis de confronter ainsi des points de vue très différents, issues de la robotique, des sciences cognitives, de l'intelligence artificielle ou de la linguistique.

- Séminaire 1 - Intelligence artificielle et modèles théoriques de l'origine du langage, Luc Steels (Bruxelles), 8 janvier 2018.

- Séminaire 2 - Apprentissage automatique non supervisé du langage: des phonèmes aux phrases, Emmanuel Dupoux (ENS Paris), 15 janvier 2018.

- Séminaire 3 - Can artificial neural networks learn compositional reasoning?, Marco Baroni (Facebook AI Paris), 22 janvier 2018.

- Séminaire 4 - Distributional semantics and vector codes for concepts and their combinations, Alessandro Lenci (Pise), 29 janvier 2018.

- Séminaire 5 - Les mots, fossiles de la pensée humaine, Mariano Sigman (Buenos Aires), 5 février 2018.

- Séminaire 6 - Avancées dans la formalisation de l'universalité des représentations linguistiques, Luigi Rizzi (Sienne, Genève), 12 février 2018. 


\section{COURS À L'EXTÉRIEUR - DES SCIENCES COGNITIVES À LA SALLE DE CLASSE}

La création du Conseil scientifique de l'Éducation nationale souligne, si besoin en était, les attentes de la société concernant les sciences cognitives et leur application à l'école. Les deux cours donnés en République dominicaine visaient à faire le point sur les contributions récentes des sciences cognitives dans le domaine de l'apprentissage, et notamment de l'apprentissage de la lecture.

\section{Sciences cognitives et éducation : les grands principes de l'apprentissage}

Université autonome de Saint-Domingue, 20 février 2018

Que signifie «apprendre»? Comme le cerveau de toutes les autres espèces, le cerveau humain apprend en modifiant ses circuits neuronaux, par le biais de modifications de ses millions de milliards de synapses - mais il met également en jeu plusieurs astuces qui font de lui le plus efficace et réactif dispositif d'apprentissage que l'on connaisse dans la nature. Les mécanismes cognitifs qu'il déploie peuvent être décrits à partir de grands piliers ou mécanismes qui modulent la capacité d'apprendre : attention, motivation, engagement actif, curiosité, retour sur erreurs, automatisation, sommeil... Ce cours a mis l'accent sur l'apport pratique des sciences cognitives à leur compréhension, dans la perspective d'améliorer les gestes pédagogiques de l'enseignant et l'art d'apprendre.

\section{Cerveau, lecture et alphabétisation}

Académie des sciences de la République dominicaine

L'apprentissage de la lecture transforme profondément le cerveau de l'enfant - mais il s'appuie sur des compétences et des circuits neuronaux préexistants, qu'il recycle. Apprendre à lire consiste à accéder aux réseaux du langage parlé par le biais de la vision. L'imagerie cérébrale, chez l'adulte comme chez l'enfant, a permis de comprendre comment ces réseaux se modifient avec l'apprentissage, avec notamment l'apparition, au sein des aires visuelles occipito-temporales de l'hémisphère gauche, d'une région spécialisée pour les mots écrits. Ces résultats de neurosciences cognitives convergent avec les résultats des recherches purement psychologiques et éducatives, et sont riches d'enseignements pour le choix des stratégies d'enseignement de la lecture à l'école.

\section{RECHERCHE}

Nous avons principalement poursuivi le projet de recherches «NeuroSyntax » financé par l'European Research Council (ERC), visant à analyser les mécanismes cérébraux de la compétence de l'espèce humaine pour la syntaxe dans tous les domaines : langue naturelle, mais aussi mathématiques et musique. Les travaux de thèse de Marie Amalric ont permis de confirmer l'existence d'un réseau cérébral systématiquement activé par la réflexion mathématique, y compris chez les mathématiciens aveugles (l'un d'entre eux aveugle de naissance). Avec Thomas Hannagan, nous avons proposé une théorie mathématique pour l'émergence précoce d'une fraction de ce réseau : le circuit responsable du codage des nombres, qui semble 
être présent dès la naissance chez le nouveau-né. Bien que le codage des nombres soit partagé avec de nombreuses autres espèces animales, nous pensons que la singularité de l'espèce humaine résiderait dans l'existence d'un « langage de la pensée » interne, combinatoire, et qui serait particulier à l'espèce humaine. Avec Marie Amalric et Liping Wang, nous avons conçu un nouveau test de cette idée, fondée sur des séquences spatiales géométriques, et nous avons montré qu'effectivement, la mémoire des séquences spatiales peut être prédite par la compacité de leur représentation dans ce langage de la pensée.

En collaboration avec Liping Wang à Shanghai et avec Zenas Chao à Tokyo, nous avons commencé à explorer les limites de la représentation des séquences visuospatiales et auditives chez le singe macaque. Nous avons analysé finement les réponses électrophysiologiques au test «local-global » et montré que le cerveau du singe macaque comprend des circuits prédictifs hiérarchiques, avec échange de signaux d'erreurs lorsque ces prédictions s'avèrent en décalage avec la réalité sensorielle. De façon surprenante, dans un article important dans Current Biology, nous avons montré que le singe macaque était capable d'apprendre une séquence spatiale " en miroir », dont la description nécessite une grammaire indépendante du contexte, et qui était donc supposée hors de portée du primate non humain.

Les nombreux travaux du laboratoire fondés sur la magnéto-encéphalographie ont permis de démontrer la remarquable capacité de suivre le déroulement temporel des opérations cognitives, par exemple toutes les étapes d'un calcul mental (thèse de Pedro Pinheiro-Chagas), ou de la sélection d'une image en mémoire de travail (travaux de Sébastien Marti). Cette dernière recherche s'inscrit dans la continuité de nos travaux antérieurs sur les mécanismes cérébraux de la prise de conscience des informations du monde extérieur. Dans ce domaine, deux articles importants ont été publiés dans Science : l'un proposant une revue des mécanismes de la conscience et l'autre, empirique, en collaboration avec l'équipe de Pieter Roelfsema, analysant leurs corrélats dans les aires V1, V4 et dans le cortex préfrontal chez le singe macaque.

Enfin, dans le domaine éducatif, l'année 2018 a vu notamment la publication d'un long travail d'IRM longitudinale chez l'enfant d'âge scolaire, avec des IRM tout au long de l'année de CP, afin de suivre les changements comportementaux et cérébraux liés à l'apprentissage de la lecture.

Nommé président du Conseil scientifique de l'Éducation nationale fin 2017, Stanislas Dehaene s'est appuyé sur ces résultats, et sur un réseau de vingt autres scientifiques volontaires, pour commencer à proposer des recommandations pédagogiques et des synthèses qui soient utiles au ministère de l'Éducation nationale.

\section{PuBLicATIONS}

AmAlRic M. et Dehaene S., « Cortical circuits for mathematical knowledge : Evidence for a major subdivision within the brain's semantic networks », Philosophical Transactions of the Royal Society of London: Series B, Biological Sciences, vol. 373, n 1740, 2018, p. 20160515 , DOI : 10.1098/rstb.2016.0515.

AMALRIC M., DENGHIEN I. et DEHAENE S., « On the role of visual experience in mathematical development : Evidence from blind mathematicians », Developmental Cognitive Neuroscience, vol. 30, 2018, p. 314-323, DOI : 10.1016/j.dcn.2017.09.007.

Barttfeld P., Abboud S., Lagercrantz H., AdÉn U., Padilla N., Edwards A.D., Cohen L., Sigman M., Dehaene S. et Dehaene-Lambertz G., « A lateral-to-mesial 
organization of human ventral visual cortex at birth », Brain Structure and Function, 2018, p. 1-13, DOI : 10.1007/s00429-018-1676-3.

Berkovitcha L., Del Cul A., Maheu M. et Dehaene S., «Impaired conscious access and abnormal attentional amplification in schizophrenia », NeuroImage : Clinical, vol. 18, 2018, p. 835-848, DOI : 10.1016/j.nicl.2018.03.010.

Chao Z.C., TAKaura K., Wang L., Fuji N. et Dehaene S., « Large-scale cortical networks for hierarchical prediction and prediction error in the primate brain », Neuron, vol. 100, $\mathrm{n}^{\circ} 5$, 2018, p. 1252-1266.e3, DOI : 10.1016/j.neuron.2018.10.004.

Dehaene-Lambertz G., Monzalvo K. et Dehaene S., « The emergence of the visual word form: Longitudinal evolution of category-specific ventral visual areas during reading acquisition », K. GRILL-SPECTOR (dir.), PLOS Biology, vol. 16, n 3, 2018, e2004103, DOI : 10.1371/journal.pbio.2004103.

DEHAENE S., « The error-related negativity, self-monitoring, and consciousness », Perspectives on Psychological Science, vol. 13, no 2, 2018, p. 161-165, DOI : 10.1177/1745691618754502.

Dotan D., Meyniel F. et DehaEnE S., «On-line confidence monitoring during decision making », Cognition, vol. 171, 2018, p. 112-121, DOI : 10.1016/j.cognition.2017.11.001.

Hannagan T., Nieder A., Viswanathan P. et DehaEnE S., « A random-matrix theory of the number sense », Philosophical Transactions of the Royal Society of London: Series B : Biological Sciences, vol. 373, no 1740, 2018, p. 20170253, DOI : 10.1098/rstb.2017.0253.

Jiang X., Long T., CaO W., Li J., Dehaene S. et Wang L., « Production of supra-regular spatial sequences by macaque monkeys », Current Biology, vol. 28, no 12, 2018, p. 1851-1859. e4, DOI : 10.1016/j.cub.2018.04.047

Kolinsky R., Morais J., COHEn L. et Dehaene S., « Les bases neurales de l'apprentissage de la lecture », Langue française, vol. 199, n 3, 2018, p. 17-33, DOI : 10.3917/lf.199.0017.

Lasne G., Piazza M., Dehaene S., Kleinschmidt A. et Eger E., «Discriminability of numerosity-evoked fMRI activity patterns in human intra-parietal cortex reflects behavioral numerical acuity », Cortex : a Journal Devoted to the Study of the Nervous System and Behavior, 2018, 198002, DOI : 10.1016/j.cortex.2018.03.008.

Nakamura K., Makuuchi M., Oga T., Mizuochi-Endo T., Iwabuchi T., NaKaJima Y. et DEHAENE S., «Neural capacity limits during unconscious semantic processing », The European Journal of Neuroscience, vol. 47, nº 8, 2018, p. 929-937, DOI : 10.1111/ejn.13890.

Pinheiro-Chagas P., Daitch A., PARVizi J. et DehaEnE S., «Brain mechanisms of arithmetic: A crucial role for ventral temporal cortex », Journal of Cognitive Neuroscience, vol. 30, no 12, 2018, p. 1757-1772, DOI : 10.1162/jocn_a_01319.

Pinheiro-Chagas P., PiAZZa M. et DehaEnE S., « Decoding the processing stages of mental arithmetic with magnetoencephalography », Cortex, vol. 114, 2019, p. 124-139, DOI : 10.1016/j.cortex.2018.07.018.

Salvador A., Berkovitch L., Vinckier F., Cohen L., Naccache L., Dehaene S. et GAILlARD R., «Unconscious memory suppression », Cognition, vol. 180, 2018, p. 191-199, DOI : 10.1016/j.cognition.2018.06.023.

Uhrig L., Sitt J.D., Jacob A., Tasserie J., Barttfeld P., Dupont M., Dehaene S. et JARRAYA B., «Resting-state dynamics as a cortical signature of anesthesia in monkeys », Anesthesiology. The Journal of the American Society of Anesthesiologists, vol. 129, $\mathrm{n}^{\circ}$ 5, 2018, p. 942-958, DOI : 10.1097/ALN.0000000000002336.

Vugt B. van, Dagnino B., Vartak D., SAFAai H., Panzeri S., Dehaene S. et ROELFSEMA P.R., « The threshold for conscious report : Signal loss and response bias in visual and frontal cortex », Science, vol. 360, no 6388, 2018, p. 537-542, DOI : 10.1126/science. aar7186. 
Amalric M., Wang L., Pica P., Figueira S., Sigman M. et Dehaene S., « The language of geometry: Fast comprehension of geometrical primitives and rules in human adults and preschoolers », PLOS Computational Biology, vol. 13, n 1, 2017, e1005273, DOI : 10.1371/ journal.pcbi.1005273.

BERKOVITCH L., DEHAENE S. et GAILlARD R., «Disruption of conscious access in schizophrenia », Trends in Cognitive Sciences, vol. 21, no 11, 2017, p. 878-892, DOI : 10.1016/j.tics.2017.08.006.

Braga L.W., Amemiya E., Tauil A., Suguieda D., Lacerda C., Klein E., DehaeneLAMBERTZ G. et DEHAENE S., «Tracking adult literacy acquisition with functional MRI: A single-case study. », Mind, Brain, and Education, vol. 11, no 3, 2017, p. 121-132, DOI : $10.1111 /$ mbe. 12143 .

DeHAENE S., LAU H. et KoUIDER S., « What is consciousness, and could machines have it? », Science, vol. 358, no 6362, 2017, p. 486-492, DOI : 10.1126/science.aan8871.

MARTI S. et DEhAENE S., «Discrete and continuous mechanisms of temporal selection in rapid visual streams », Nature Communications, vol. 8, $\mathrm{n}^{\mathrm{0}}$ 1, 2017, p. 1955, DOI : 10.1038/ s41467-017-02079-X.

MEYNIEL F. et DEHAENE S., «Brain networks for confidence weighting and hierarchical inference during probabilistic learning », Proceedings of the National Academy of Sciences of the United States of America, vol. 114, $\mathrm{n}^{\mathrm{o}}$ 19, 2017, E3859-E3868, DOI : 10.1073/ pnas. 1615773114.

Mongelli V., Dehaene S., Vinckier F., Peretz I., Bartolomeo P. et Cohen L., « Music and words in the visual cortex : The impact of musical expertise », Cortex, vol. 86, 2017, Is a « single » brain model sufficient?, p. 260-274, DOI : 10.1016/j.cortex.2016.05.016.

Moreno A., Limousin F., Dehaene S. et Pallier C., « Brain correlates of constituent structure in sign language comprehension », NeuroImage, vol. 167, 2018, p. 151-161, DOI : 10.1016/j.neuroimage.2017.11.040.

Nelson M.J., Karoui I.E., Giber K., Yang X., Cohen L., Koopman H., Cash S.S., NACCAChe L., Hale J.T., PAllier C. et DehaEne S., « Neurophysiological dynamics of phrase-structure building during sentence processing », Proceedings of the National Academy of Sciences of the United States of America, vol. 114, $\mathrm{n}^{\mathrm{O}}$ 18, 2017, E3669-E3678, DOI : 10.1073/pnas.1701590114.

Pinheiro-Chagas P., Dotan D., Piazza M. et Dehaene S., « Finger tracking reveals the covert stages of mental arithmetic », Open Mind. Discoveries in Cognitive Science, vol. 1, n 1 1, 2017, p. 30-41, DOI : 10.1162/OPMI_a_00003.

TrÜButscheK D., Marti S., OJeda A., King J.-R., Mi Y., TsodyKs M. et Dehaene S., « A theory of working memory without consciousness or sustained activity », ELife, vol. 6, 2017, e23871, DOI : 10.7554/eLife.23871. 
